
Shaohui Wang

\title{
Least eigenvalue of the connected graphs whose complements are cacti
}

https://doi.org/10.1515/math-2019-0097

Received November 6, 2018; accepted August 27, 2019

Abstract: Suppose that $\Gamma$ is a graph of order $n$ and $A(\Gamma)=\left[a_{i, j}\right]$ is its adjacency matrix such that $a_{i, j}$ is equal to 1 if $v_{i}$ is adjacent to $v_{j}$ and $a_{i, j}$ is zero otherwise, where $1 \leq i, j \leq n$. In a family of graphs, a graph is called minimizing if the least eigenvalue of its adjacency matrix is minimum in the set of the least eigenvalues of all the graphs. Petrović et al. [On the least eigenvalue of cacti, Linear Algebra Appl., 2011, 435, 2357-2364] characterized a minimizing graph in the family of all cacti such that the complement of this minimizing graph is disconnected. In this paper, we characterize the minimizing graphs $G \in \Omega_{n}^{c}$, i.e.

$$
\lambda_{\min }(G) \leq \lambda_{\min }\left(C^{c}\right)
$$

for each $C^{c} \in \Omega_{n}^{c}$, where $\Omega_{n}^{c}$ is a collection of connected graphs such that the complement of each graph of order $n$ is a cactus with the condition that either its each block is only an edge or it has at least one block which is an edge and at least one block which is a cycle.

Keywords: adjacency matrix, least eigenvalue, connected graphs, cacti

MSC: 15A18, 05C50, 05C40, 05D05

\section{Introduction}

Let $\Gamma=(V(\Gamma), E(\Gamma))$ be a graph such that $V(\Gamma)=\left\{v_{i}: 1 \leq i \leq n\right\}$ and $E(\Gamma)$ are set of vertices and edges respectively. Assume that all the considered graphs are simple, finite and undirected. For each $i$, the degree $d(i)$ is the number of incident edges on $v_{i}$. The adjacency matrix of $\Gamma$ is $A(\Gamma)=\left[a_{i, j}\right]$ with $a_{i, j}$ equal to 1 if $v_{i}$ is linked to $v_{j}$ and $a_{i, j}$ is zero for the rest case, where $1 \leq i, j \leq n$. The solutions of $\operatorname{det}(A(\Gamma)-\lambda I)=0$ are eigenvalues of $\Gamma$. It is interesting to note that $A(\Gamma)$ is always symmetric and real, all the eigenvalues can be arrange as $\lambda_{1}(\Gamma) \leq \lambda_{2}(\Gamma) \leq \ldots \leq \lambda_{n}(\Gamma)$. The eigenvectors corresponding to the least eigenvalue $\lambda_{1}(\Gamma)$ and the greatest eigenvalue (spectral radius) $\lambda_{n}(\Gamma)$ are called first eigenvector (FEv) and Perron-vector respectively.

The spectrum of the adjacency matrix for an undirected graph is first time studied by Collatz and Sinogowitz (1957), see [1]. Later on, many researchers discussed the largest eigenvalue (spectral radius) in the area of spectra of graphs, see [2, 3]. It is observed that the least eigenvalue did not receive the attention of researchers as compare of the largest eigenvalue. From the few of results of the least eigenvalues on the graphs, the bounds related results can be found in $[4,5]$. For further study, we refer [6-13]. A graph $\Gamma$ is said

${ }^{\star}$ Corresponding Author: Haiying Wang: School of Science, China University of Geosciences (Beijing), Beijing, P.R. China; E-mail: whycht@126.com

Muhammad Javaid, Sana Akram: Department of Mathematics, School of Science, University of Management and Technology, Lahore 54770, Pakistan; E-mail: javaidmath@gmail.com, sanaakram.umt@gmail.com

Muhammad Jamal: Department of Mathematics and Statistics, PMAS-Arid Agriculture University, Rawalpindi, Pakistan; E-mail: jamal@uaar.edu.pk

Shaohui Wang: Department of Mathematics, Louisiana College, Pineville, LA 71359, USA; E-mail: shaohuiwang@yahoo.com 
to be minimizing in a certain collection of graphs, if the least eigenvalue of $A(\Gamma)$ is minimum in the set of all the least eigenvalues of the other graphs in the same collection.

Let $\mathcal{G}(p, q)$ be a collection of connected graphs in which each graph is of $p$ order and $q$ size such that $0<q<\frac{p(p-1)}{2}$. The minimizing graph in $\mathcal{G}(p, q)$ characterized by Bell et al. [14] is stated in the below result:

Theorem 1.1. Minimizing graph in $\mathcal{G}(p, q)$ is either a join of two nested split graphs, or a bipartite graph.

It is important to note that the complements of the graphs characterized by Bell contain the cliques such that order of each clique is greater or equal to $\frac{p}{2}$ or these are disconnected. After it, the question is raised to investigate the minimizing graphs in the collection of connected graphs such that the complement of each graph contains the cliques of small sizes. Motivated by it, the minimizing graph in the collection of connected graphs such that the complement of each graph is trees, unicyclic or bicyclic are characterized by Fan, Zhang, Wang, Li and Javaid, see [15-18]. For further study, we refer to [19-22]. In this paper, the minimizing graph is characterized in the collection of connected graphs such that the complement of each graph is cactus with the condition that each block of a cactus of order $n$ is only an edge or a cactus of order $n$ has at least one block which is an edge and at least one block which is a cycle. In the rest of paper; Section 2 includes some basic definitions and terminologies, Section 3 contains the proofs of some important lemmas and Section 4 has the main results in which minimizing graph is characterized in the family of connected graphs with the condition that the complement of each graph is cactus.

\section{Preliminaries}

A connected graph is called cactus if and only if its every block is either a simple cycle or a single edge. A cactus is a tree if and only if its each block is an edge. An edge of a cactus is a cycle edge if it is in some cycle, and tree edge, otherwise. A cactus is said to be a bundle if there is a single common vertex on all of its cycles. Let $B_{1}(n)$ be the bundle of order $n+1$ obtained from a star $K_{1, n}$ of the same order with central vertex $V_{0}$ by adding the edges $v_{i} v_{i+1}$, where $i \in\{1,3,5, \ldots, n-1\}$ and $n=0 \bmod (2)$. Thus, the central vertex $v_{0}$ of the bundle $B_{1}(n)$ has degree $n$ and each remaining vertex is of degree 2. Similarly, let $B_{2}(n)$ be a bundle obtained from $K_{1, n}$ by adding the edges $v_{i} v_{i+1}$, where $i \in\{1,3,5, \ldots, n-2\}, n=1 \bmod (2)$ and $\left|V\left(B_{2}(n)\right)\right|=n+1$. Thus, for the vertices of $B_{2}(n), d\left(v_{0}\right)=n, d\left(v_{n}\right)=1$ and $d\left(v_{i}\right)=2$, where $1 \leq i \leq n-1$.

We define some particular cacti which are obtained from the aforesaid bundles.

Definition 2.1. Assume that $p, q=0 \bmod (2)$ are positive integers. Let $B_{1}(p)$ and $B_{1}(q)$ be two bundles. The cactus graph $C_{1}(p, q)$ is constructed by the join of a vertex of $B_{1}(p)$ with a vertex $B_{1}(q)$, where both the vertices are of degree 2. Thus, $V\left(C_{1}(p, q)\right)=\left\{v_{1}^{j}: 1 \leq j \leq p-2\right\} \cup\left\{v_{j}: 2 \leq j \leq 7\right\} \cup\left\{v_{8}^{j}: 1 \leq j \leq q-2\right\}$ and $E\left(C_{1}(p, q)\right)=\left\{v_{2} v_{1}^{j}: 1 \leq j \leq p-2\right\} \cup\left\{v_{2} v_{3}, v_{2} v_{4}\right\} \cup\left\{v_{1}^{j} v_{1}^{j+1}: j=1,3,5, \ldots, p-3\right\} \cup\left\{v_{j} v_{j+1}: 3 \leq j \leq\right.$ $5\} \cup\left\{v_{7} v_{5}, v_{7} v_{6}\right\} \cup\left\{v_{7} v_{8}^{j}: 1 \leq j \leq q-2\right\} \cup\left\{v_{8}^{j} v_{8}^{j+1}: j=1,3,5, \ldots, q-3\right\}$ with $\left|C_{1}(p, q)\right|=2+p+q=n$.

Assume that $p, q \equiv 1 \bmod (2)$ and $p, q \geq 3$. If a vertex of the bundle $B_{2}(p)$ is joined with a vertex of the bundle $B_{2}(q)$ then we obtain the cactus graph $C_{1}^{\prime}(p, q)$, where both the chosen vertices are pendent and $n=2+p+q=\left|C_{1}^{\prime}(p, q)\right|$. For $p \geq 3, q \geq 2, p \equiv 1 \bmod (2)$ and $q \equiv 0 \bmod (2)$, if we join a vertex of the bundle $B_{2}(p)$ to a vertex of the bundle $B_{1}(q)$ then we obtain a cactus graph $C_{2}(p, q)$, where the chosen vertices are of degree 1 and 2 respectively and $n=2+p+q=\left|C_{2}(p, q)\right|$. Similarly, if we assume $p \geq 2, q \geq 3, p=0 \bmod (2)$ and $q=1 \bmod (2)$, and choose two vertices of degree 2 and 1 in $B_{1}(p)$ and $B_{2}(q)$ respectively. On joining these chosen vertices by an edge, we obtain the cactus graph $C_{2}^{\prime}(p, q)$ with $n=2+p+q=\left|C_{1}^{\prime}(p, q)\right|$.

We note that $C_{1}(p, q) \cong C_{1}(q, p)$ and $C_{1}^{\prime}(p, q) \cong C_{1}^{\prime}(q, p)$ as $p$ and $q$ both are even in $C_{1}(p, q)$ and odd in $C_{1}^{\prime}(p, q)$. Moreover, as $p$ is odd and $q$ is even in $C_{2}(p, q)$, and $p$ is even and $q$ is odd in $C_{2}^{\prime}(p, q)$ therefore $C_{2}(p, q) \cong C_{2}(q, p), C_{2}^{\prime}(p, q) \cong C_{2}^{\prime}(q, p)$ and $C_{2}(q, p) \cong C_{2}^{\prime}(p, q)$. The cacti $C_{1}(p, q)$ and $C_{1}^{\prime}(p, q)$ are presented in Figure 1((a) and (b)) and the cacti $C_{2}(p, q)$ and $C_{2}^{\prime}(p, q)$ are presented in Figure 2((a) and (b)).

Let $\Omega_{1, n}$ be the class of cacti other than stars such that each block of a cactus is an edge and $\Omega_{2, n}$ be a class of cacti other than bundles such that at least one block of each cactus is an edge and at least one 


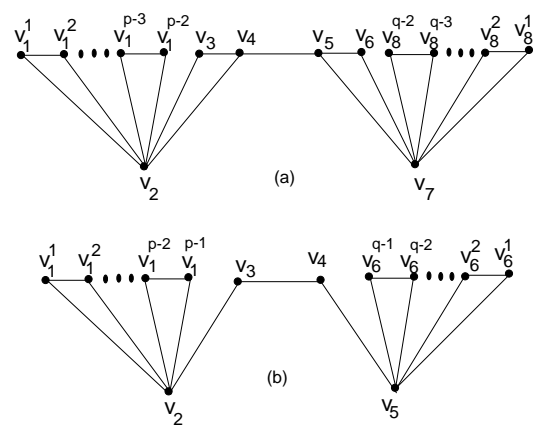

Figure 1: (a) $C_{1}(p, q)$ and (b) $C_{1}^{\prime}(p, q)$.

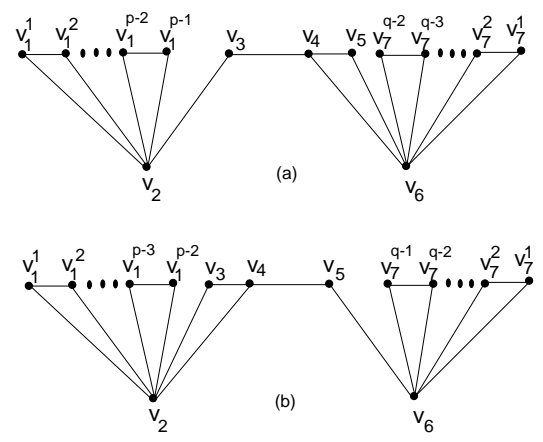

Figure 2: (a) $C_{2}(p, q)$ and (b) $C_{2}^{\prime}(p, q)$.

block is a cycle. Let $\Omega_{n}$ be a class of cacti other than stars and bundles such that either all the blocks of a cactus are edges or a cactus has at least one block which is a cycle and at least one block which is an edge, i.e. $\Omega_{n}=\Omega_{1, n} \cup \Omega_{2, n}$. Thus, we obatain $\Omega_{n}^{c}=\left\{\Gamma^{c}: \Gamma^{c}\right.$ is connected, $\left.\left|\Gamma^{c}\right|=n \wedge \Gamma \in \Omega_{n}\right\}$. By interlacing theorem, $\lambda_{\min }(\Gamma) \leq-1$ if $\Gamma$ contains at least one edge. Moreover, equality holds if $\Gamma$ is a complete graph. Another way to achieve this equality is if $\Gamma=\cup_{i} G_{i}$, where all $G_{i}$ are complete graphs and at least one $G_{i}$ is non-trivial. Thus, for $\Gamma \in \Omega_{n}, \lambda_{\min }\left(\Gamma^{c}\right)<-1$.

If $\phi^{\prime}: V(\Gamma) \rightarrow\left\{X_{i}: 1 \leq i \leq n\right\}$ is a 1-1 map such that $\phi^{\prime}\left(u_{i}\right)=X_{i}$ for each $u_{i} \in V(\Gamma)$ then it is said to be defined on the graph $\Gamma$. The eigenvector $X$ of $A(\Gamma)$ is naturally defined on $V(\Gamma)$. Thus, we have

$$
X^{T} A X=2 \sum_{u v \in E(\Gamma)} X_{u} X_{v}
$$

The eigenequation for each $v \in V(\Gamma)$ is

$$
\lambda X_{v}=\sum_{u \in N_{\Gamma}(v)} X_{u}
$$

where all adjacent to $v$ are in $N_{\Gamma}(v)$. If $X \in \mathrm{R}^{n}$ is an arbitrary unit vector, we have

$$
\lambda_{\min }(\Gamma) \leq X^{T} A(\Gamma) X,
$$

where equality holds iff $X$ is a FEv. If $\Gamma^{c}$ is complement of $\Gamma$, then $A\left(\Gamma^{c}\right)=J-I-A(\Gamma)$ with $J$ and $I$ as all-ones and identity matrix respectively. Thus, for $X \in \mathrm{R}^{n}$

$$
X^{T} A\left(\Gamma^{c}\right) X=X^{T}(J-I) X-X^{T} A(\Gamma) X .
$$


Let $Y_{1}$ be FEv of $C_{1}(p, q)^{c}$ which is defined on it. By (2.2), the vertices $v_{1}^{j}$ for $1 \leq j \leq p-2, v_{2}, v_{3}, v_{4}, v_{5}$, $v_{6}, v_{7}$ and $v_{8}^{j}$ for $1 \leq j \leq q-2$ having values in $Y_{1}$, say $X_{j}$ for $1 \leq j \leq 8$ respectively. If $\lambda_{\min }\left(C_{1}(p, q)^{c}\right)=\lambda_{1}$ then

$$
\left\{\begin{array}{l}
\lambda_{1} X_{1}=(p-4) X_{1}+X_{3}+X_{4}+X_{5}+X_{6}+X_{7}+(q-2) X_{8}, \\
\lambda_{1} X_{2}=X_{5}+X_{6}+X_{7}+(q-2) X_{8}, \\
\lambda_{1} X_{3}=(p-2) X_{1}+X_{5}+X_{6}+X_{7}+(q-2) X_{8}, \\
\lambda_{1} X_{4}=(p-2) X_{1}+X_{6}+X_{7}+(q-2) X_{8}, \\
\lambda_{1} X_{5}=(p-2) X_{1}+X_{2}+X_{3}+(q-2) X_{8}, \\
\lambda_{1} X_{6}=(p-2) X_{1}+X_{2}+X_{3}+X_{4}+(q-2) X_{8}, \\
\lambda_{1} X_{7}=(p-2) X_{1}+X_{2}+X_{3}+X_{4}, \\
\lambda_{1} X_{8}=(p-2) X_{1}+X_{2}+X_{3}+X_{4}+X_{5}+X_{6}+(q-4) X_{8} .
\end{array}\right.
$$

Take $Y_{1}=\left(X_{1}, X_{2}, X_{3}, X_{4}, X_{5}, X_{6}, X_{7}, X_{8}\right)^{T}$ then the matrix equation is $\left(A-\lambda_{1} I\right) Y_{1}=0$ and

$$
\begin{aligned}
f_{1}(\lambda, p, q)=\operatorname{det}(A-\lambda I)= & (4+p q-2 p-2 q)+(16-4 p-4 q) \lambda+(-4-7 p q+12 p+12 q) \lambda^{2} \\
& +(-48+2 p q+14 p+14 q) \lambda^{3}+(-20+7 p q-9 p-9 q) \lambda^{4} \\
& +(24+2 p q-16 p-16 q) \lambda^{5}+(24-7 p-7 q) \lambda^{6}+(8-p-q) \lambda^{7}+\lambda^{8} \ldots
\end{aligned}
$$

with least root $\lambda_{1}$.

Let $Y_{1}^{\prime}$ be FEv of $C_{1}^{\prime}(p, q)^{c}$. By (2.2), the vertices $v_{1}^{j}$ for $1 \leq j \leq p-1, v_{2}, v_{3}, v_{4}, v_{5}$ and $v_{6}^{j}$ for $1 \leq j \leq q-1$ having values in $Y_{1}^{\prime}$, say $X_{j}$ for $1 \leq j \leq 6$ respectively. If $\lambda_{\min }\left(C_{1}^{\prime}(p, q)^{c}\right)=\lambda_{1}^{\prime}$ then

$$
\left\{\begin{array}{l}
\lambda_{1}^{\prime} X_{1}=(p-3) X_{1}+X_{3}+X_{4}+X_{5}+(q-1) X_{6}, \\
\lambda_{1}^{\prime} X_{2}=X_{4}+X_{5}+(q-1) X_{6}, \\
\lambda_{1}^{\prime} X_{3}=(p-1) X_{1}+X_{5}+(q-1) X_{6}, \\
\lambda_{1}^{\prime} X_{4}=(p-1) X_{1}+X_{2}+(q-1) X_{6}, \\
\lambda_{1}^{\prime} X_{5}=(p-1) X_{1}+X_{2}+X_{3}, \\
\lambda_{1}^{\prime} X_{6}=(p-1) X_{1}+X_{2}+X_{3}+X_{4}+(q-1) X_{6} .
\end{array}\right.
$$

Take $Y_{1}^{\prime}=\left(X_{1}, X_{2}, X_{3}, X_{4}, X_{5}, X_{6}\right)^{T}$ then the matrix equation is $\left(A-\lambda_{1}^{\prime} I\right) Y_{1}^{\prime}=0$ and

$$
\begin{aligned}
f_{1}^{\prime}(\lambda, p, q)= & \operatorname{det}(A-\lambda I) \\
= & (8-2 p-2 q)+(-8-2 p q+7 p+7 q) \lambda+(-18+3 p q+2 p+2 q) \lambda^{2}+(2 p q-7 p-7 q) \lambda^{3} \\
& +(11-5 p-5 q) \lambda^{4}+(6-p-q) \lambda^{5}+\lambda^{6} \ldots
\end{aligned}
$$

with least root $\lambda_{1}^{\prime}$.

Let $Y_{2}$ be FEv of $C_{2}(p, q)^{c}$. By (2.2), the vertices $v_{1}^{j}$ for $1 \leq i \leq p-1, v_{2}, v_{3}, v_{4}, v_{5}, v_{6}$, and $v_{7}^{j}$ for $1 \leq j \leq q-2$ having values in $Y_{2}$, say $X_{j}$ for $1 \leq j \leq 7$ respectively. If $\lambda_{\min }\left(C_{2}(p, q)^{c}\right)=\lambda_{2}$ then

$$
\left\{\begin{array}{l}
\lambda_{2} X_{1}=(p-3) X_{1}+X_{3}+X_{4}+X_{5}+X_{6}+(q-2) X_{7} \\
\lambda_{2} X_{2}=X_{4}+X_{5}+X_{6}+(q-2) X_{7} \\
\lambda_{2} X_{3}=(p-1) X_{1}+X_{5}+X_{6}+(q-2) X_{7} \\
\lambda_{2} X_{4}=(p-1) X_{1}+X_{2}+(q-2) X_{7} \\
\lambda_{2} X_{5}=(p-1) X_{1}+X_{2}+X_{3}+(q-2) X_{7} \\
\lambda_{2} X_{6}=(p-1) X_{1}+X_{2}+X_{3} \\
\lambda_{2} X_{7}=(p-1) X_{1}+X_{2}+X_{3}+X_{4}+X_{5}+(q-4) X_{7}
\end{array}\right.
$$

Take $Y_{2}=\left(X_{1}, X_{2}, X_{3}, X_{4}, X_{5}, X_{6}, X_{7}\right)^{T}$ then the matrix equation is $\left(A-\lambda_{2} I\right) Y_{2}=0$ and

$$
\begin{aligned}
f_{2}(\lambda, p, q)= & \operatorname{det}(A-\lambda I) \\
= & (-6-p q+2 p+3 q)+(-8-2 p-2 q+3 p q) \lambda+(26+p q-10 p-13 q) \lambda^{2}+(23-5 p q+3 p+q) \lambda^{3} \\
& +(-9-2 p q+11 p+11 q) \lambda^{4}+(-17+6 p+6 q) \lambda^{5}+(-7+p+q) \lambda^{6}-\lambda^{7} \ldots
\end{aligned}
$$


with least root $\lambda_{2}$.

Let $Y_{2}^{\prime}$ be the FEv of $C_{2}^{\prime}(p, q)^{c}$. By (2.2), the vertices $v_{1}^{j}$ for $1 \leq j \leq p-2, v_{2}, v_{3}, v_{4}, v_{5}, v_{6}$, and $v_{7}^{j}$ for $1 \leq j \leq q-1$ having values in $Y_{2}^{\prime}$, say $X_{j}$ for $1 \leq j \leq 7$ respectively. If $\lambda_{\min }\left(C_{2}^{\prime}(p, q)^{c}\right)=\lambda_{2}$ then

$$
\left\{\begin{array}{l}
\lambda_{2}^{\prime} X_{1}=(p-4) X_{1}+X_{3}+X_{4}+X_{5}+X_{6}+(q-1) X_{7}, \\
\lambda_{2}^{\prime} X_{2}=X_{5}+X_{6}+(q-1) X_{7}, \\
\lambda_{2}^{\prime} X_{3}=(p-2) X_{1}+X_{5}+X_{6}+(q-1) X_{7}, \\
\lambda_{2}^{\prime} X_{4}=(p-2) X_{1}+X_{6}+(q-1) X_{7}, \\
\lambda_{2}^{\prime} X_{5}=(p-2) X_{1}+X_{2}+X_{3}+(q-1) X_{7}, \\
\lambda_{2}^{\prime} X_{6}=(p-2) X_{1}+X_{2}+X_{3}+X_{4}, \\
\lambda_{2}^{\prime} X_{7}=(p-2) X_{1}+X_{2}+X_{3}+X_{4}+X_{5}+(q-3) X_{7} .
\end{array}\right.
$$

Take $Y_{2}^{\prime}=\left(X_{1}, X_{2}, X_{3}, X_{4}, X_{5}, X_{6}, X_{7}\right)^{T}$, the matrix equation is $\left(A-\lambda_{2}^{\prime} I\right) Y_{2}^{\prime}=0$ and

$$
\begin{aligned}
f_{2}^{\prime}(\lambda, p, q)= & \operatorname{det}(A-\lambda I) \\
= & (-6-p q+2 p+3 q)+(-8-2 p-2 q+3 p q) \lambda+(26+p q-13 p-10 q) \lambda^{2}+(23-5 p q+p+3 q) \lambda^{3} \\
& +(-9-2 p q+11 p+11 q) \lambda^{4}+(-17+6 p+6 q) \lambda^{5}+(-7+p+q) \lambda^{6}-\lambda^{7} \ldots
\end{aligned}
$$

with least $\operatorname{root} \lambda_{2}^{\prime}$.

\section{Minimizing graphs}

Now, we present some important lemmas of the minimizing graph which are frequently used in next section. The classes of cacti which have graphs of even order are discussed from Lemma 3.1 to Lemma 3.6. Moreover, the cacti of odd order are studied from Lemma 3.7 to Lemma 3.10.

Firstly, we discuss the classes of cacti which have graphs of even order.

Lemma 3.1. Suppose that $p, q \geq 4, n \geq 12$ are integers with $p, q, n \equiv 0(\bmod 2)$. If $p>q+2$, then

$$
\lambda_{\min }\left(C_{1}(p-2, q+2)^{c}\right)<\lambda_{\min }\left(C_{1}(p, q)^{c}\right),
$$

where $p+q+2=n=\left|V\left(C_{1}(p-2, q+2)^{c}\right)\right|=\left|V\left(C_{1}(p, q)^{c}\right)\right|$.

Proof. From equation (2.5), we have $f_{1}(-3, p, q)=325-17(p+q)-35 p q$. Since for $p, q \geq 4 f_{1}(-3, p, q)<0$. Therefore, least root of $f_{1}(\lambda, p, q)$ is $\lambda_{1}<-3$. Moreover, $f_{1}(\lambda, p-2, q+2)=(p q-4 q)+(16-4 p-4 q) \lambda+(24-$ $2 p+26 q-7 p q) \lambda^{2}+(-56+18 p+10 q+2 p q) \lambda^{3}+(-48+5 p-23 q+7 p q) \lambda^{4}+(16-12 p-20 q+2 p q) \lambda^{5}+$ $(24-7 p-7 q) \lambda^{6}+(8-p-q) \lambda^{7}+\lambda^{8}$, and

$$
\begin{aligned}
f_{1}(\lambda, p, q)-f_{1}(\lambda, p-2, q+2) & =-2(p-q-2)\left(2 \lambda^{5}+7 \lambda^{4}+2 \lambda^{3}-7 \lambda^{2}+1\right) \\
& =-2(p-q-2)\left(\lambda-\frac{1}{2}\right)\left(\lambda+\frac{3+\sqrt{5}}{2}\right)\left(\lambda+\frac{3-\sqrt{5}}{2}\right)\left(\lambda+\frac{1+\sqrt{5}}{2}\right)\left(\lambda+\frac{1-\sqrt{5}}{2}\right) .
\end{aligned}
$$

As $p$ is greater than $q+2$ and $\lambda$ is less than -3 therefore $f_{1}(\lambda, p, q)-f_{1}(\lambda, p-2, q+2)>0$. Also, $f_{1}(-3, p-2, q+2)<0$ which implies that $\lambda_{\min }\left(C_{1}(p-2, q+2)^{c}\right)<\lambda_{\min }\left(C_{1}(p, q)^{c}\right)$.

Corollary 3.2. Suppose that $p, q \geq 4, n \geq 12$ are integers with $p, q, n \equiv 0(\bmod 2)$. If $q>p+2$, then $\lambda_{\text {min }}\left(C_{1}(p+2, q-2)^{c}\right)<\lambda_{\text {min }}\left(C_{1}(p, q)^{c}\right)$, where $p+q+2=n$.

Proof. Since, $C_{1}(p, q)^{c} \cong C_{1}(q, p)^{c}$, therefore proof is same as of Lemma 3.1.

Lemma 3.3. Suppose that $p, q \geq 4$ are integers with $p, q \equiv 0(\bmod 2)$ and $p+q+2=n=\left|V\left(C_{1}\left(\frac{n-2}{2}, \frac{n-2}{2}\right)^{c}\right)\right|=$ $\left|V\left(C_{1}(p, q)^{c}\right)\right|=\left|V\left(C_{1}\left(\frac{n}{2}, \frac{n-4}{2}\right)^{c}\right)\right|$. Then

$$
\lambda_{\text {min }}\left(C_{1}(p, q)^{c}\right) \geq\left\{\begin{array}{l}
\lambda_{\min }\left(C_{1}\left(\frac{n-2}{2}, \frac{n-2}{2}\right)^{c}\right) \text { if } n \equiv 2(\bmod 4) ; \\
\lambda_{\text {min }}\left(C_{1}\left(\frac{n}{2}, \frac{n-4}{2}\right)^{c}\right) \quad \text { if } n \equiv 0(\bmod 4) ;
\end{array}\right.
$$


where equality holds iff $p=\frac{n-2}{2}=q$ with $n \geq 14$ and $p=\frac{n}{2}$ and $q=\frac{n-4}{2}$ with $n \geq 12$.

Proof. When $n \equiv 2(\bmod 4)$, then for $p=\frac{n-2}{2}=q$, the equation (2.5) becomes $f_{1}\left(-3, \frac{n-2}{2}, \frac{n-2}{2}\right)=-(n-7.2)(n+$ 5.1428). For $n \geq 14$, we have $f_{1}\left(-3, \frac{n-2}{2}, \frac{n-2}{2}\right)<0$. (b) When $n \equiv 0(\bmod 4)$, then for $p=\frac{n}{2}$ and $q=\frac{n-4}{2}$, the equation (2.5) becomes $f_{1}\left(-3, \frac{n}{2}, \frac{n-4}{2}\right)=-(n-7.5159)(n+5.4588)$. For $n \geq 12$, we have $f_{1}\left(-3, \frac{n}{2}, \frac{n-4}{2}\right)<0$. Thus, from both the cases least root of $f_{1}(\lambda, p, q)$ is $\lambda_{1}<-3$.

Now, by Lemma 3.1, if $q+2<p$ and $\lambda<-3$, then $\lambda_{\min }\left(C_{1}(p-2, q+2)^{c}\right)<\lambda_{\min }\left(C_{1}(p, q)^{c}\right)$ and by Corollary 3.1, if $q>p+2$ and $\lambda<-3$, then $\lambda_{\text {min }}\left(C_{1}(p+2, q-2)^{c}\right)<\lambda_{\text {min }}\left(C_{1}(p, q)^{c}\right)$.

Consequently, for $n \geq 14$ and $n \equiv 2(\bmod 4)$, we have $\lambda_{\min }\left(C_{1}\left(\frac{n-2}{2}, \frac{n-2}{2}\right)^{c}\right) \leq \lambda_{\min }\left(C_{1}(p, q)^{c}\right)$ with equality iff $p=\frac{n-2}{2}=q$, and (b) for $n \geq 12$ and $n \equiv 0(\bmod 4)$, we have $\lambda_{\min }\left(C_{1}\left(\frac{n}{2}, \frac{n-4}{2}\right)^{c}\right) \leq \lambda_{\min }\left(C_{1}(p, q)^{c}\right)$ with equality iff $p=\frac{n}{2}$ and $q=\frac{n-4}{2}$.

Lemma 3.4. Suppose that $p \geq 5, q \geq 3, n \geq 12$ are integers with $p, q \equiv 1(\bmod 2)$ and $n \equiv 0(\bmod 2)$. If $p>q+2$, then

$$
\lambda_{\min }\left(C_{1}^{\prime}(p-2, q+2)^{c}\right)<\lambda_{\min }\left(C_{1}^{\prime}(p, q)^{c}\right),
$$

where $p+q+2=n$ is cardinality of both the cacti.

Proof. By (2.8), $f_{1}^{\prime}(-3, p, q)=32+(p+q)-21(p q-p-q)$. Since, for $p \geq 5$ and $q \geq 3, f_{1}^{\prime}(-3, p, q)<0$. Therefore, least root $f_{1}^{\prime}(\lambda, p, q)$ is $\lambda_{1}<-3$. Also,

$$
\begin{gathered}
f_{1}^{\prime}(\lambda, p-2, q+2)=(8-2 p-2 q)+(-2 p q+3 p+11 q) \lambda+(-30+3 p q+8 p-4 q) \lambda^{2} \\
+(2 p q-3 p-11 q-8) \lambda^{3}+(11-5 p-5 q) \lambda^{4}+(6-p-q) \lambda^{5}+\lambda^{6} \\
\begin{aligned}
f_{1}^{\prime}(\lambda, p, q)-f_{1}^{\prime}(\lambda, p-2, q+2) & =(8-4 p+4 q) \lambda^{3}+(12-6 p+6 q) \lambda^{2}+(-8+4 p-4 q) \\
& =-2 \lambda(\lambda+2)(\lambda-0.5) .
\end{aligned}
\end{gathered}
$$

Since $p$ is greater than $q+2$ and $\lambda$ is less than $-3, f_{1}^{\prime}(\lambda, p, q)-f_{1}^{\prime}(\lambda, p-2, q+2)>0$. Also, $f_{1}^{\prime}(-3, p-2, q+2)<0$ which implies that $\lambda_{\min }\left(C_{1}^{\prime}(p-2, q+2)^{c}\right)<\lambda_{\min }\left(C_{1}^{\prime}(p, q)^{c}\right)$.

Corollary 3.5. Suppose that $p \geq 3, q \geq 5, n \geq 12$ are integers with $p, q \equiv 1(\bmod 2)$ and $n \equiv 0(\bmod 2)$. If $q>p+2$, then $\lambda_{\text {min }}\left(C_{1}^{\prime}(p+2, q-2)^{c}\right)<\lambda_{\text {min }}\left(C_{1}^{\prime}(p, q)^{c}\right)$, where $p+q+2=n$.

Proof. Since, $C_{1}^{\prime}(p, q)^{c} \cong C_{1}^{\prime}(q, p)^{c}$, therefore proof is same as of Lemma 3.4.

Lemma 3.6. Suppose that $p, q \geq 3$ are integers with $p, q \equiv 1(\bmod 2)$ and $p+q+2=n=\left|V\left(C_{1}^{\prime}\left(\frac{n-2}{2}, \frac{n-2}{2}\right)^{c}\right)\right|=$ $\left|V\left(C_{1}(p, q)^{c}\right)\right|=\left|V\left(C_{1}\left(\frac{n}{2}, \frac{n-4}{2}\right)^{c}\right)\right|$. Then,

$$
\lambda_{\text {min }}\left(C_{1}^{\prime}(p, q)^{c}\right) \geq \begin{cases}\lambda_{\min }\left(C_{1}^{\prime}\left(\frac{n}{2}, \frac{n-4}{2}\right)^{c}\right) & \text { if } n \equiv 2(\bmod 4) \\ \lambda_{\min }\left(C_{1}\left(\frac{n}{2}, \frac{n-4}{2}\right)^{c}\right) & \text { if } n \equiv 0(\bmod 4) ;\end{cases}
$$

where equality holds iff $p=\frac{n}{2}$ and $q=\frac{n-4}{2}$ with $n \geq 14$, and $p=\frac{n-2}{2}=q$ with $n \geq 12$.

Proof. When $n \equiv 2(\bmod 4)$, then for $p=\frac{n}{2}$ and $q=\frac{n-4}{2}$ the equation $(2.8)$ becomes $f_{1}^{\prime}\left(-3, \frac{n}{2}, \frac{n-2}{2}\right)=-\frac{1}{4}(n-$ $6.4842)(n+0.2937)$. For $n \geq 14$, we have $f_{1}\left(-3, \frac{n}{2}, \frac{n-2}{2}\right)<0$. (b) When $n \equiv 0(\bmod 4)$, then for $p=\frac{n-2}{2}=q$ the equation (2.8) becomes $f_{1}^{\prime}\left(-3, \frac{n-2}{2}, \frac{n-2}{2}\right)=-\frac{1}{4}(n-7.3333)(n-0.8571)$. For $n \geq 12$, we have $f_{1}^{\prime}\left(-3, \frac{n-2}{2}, \frac{n-2}{2}\right)<$ 0 . Thus, from both the cases least root of $f_{1}^{\prime}(\lambda, p, q)$ is $\lambda_{1}^{\prime}<-3$.

Now, by Lemma 3.4, for $p>q+2$ and $\lambda<-3, \lambda_{\min }\left(C_{1}^{\prime}(p-2, q+2)^{c}\right)<\lambda_{\min }\left(C_{1}^{\prime}(p, q)^{c}\right)$ and by Corollary 3.5, if $q>p+2$ and $\lambda<-3$, then $\lambda_{\text {min }}\left(C_{1}^{\prime}(p+2, q-2)^{c}\right)<\lambda_{\min }\left(C_{1}^{\prime}(p, q)^{c}\right)$.

Consequently, for $n \geq 14$ and $n \equiv 2(\bmod 4)$, we have $\lambda_{\min }\left(C_{1}^{\prime}\left(\frac{n}{2}, \frac{n-4}{2}\right)^{c}\right) \leq \lambda_{\min }\left(C_{1}^{\prime}(p, q)^{c}\right)$ with equality iff $p=\frac{n}{2}$ and $q=\frac{n-4}{2}$, and (b) for $n \geq 12$ and $n \equiv 0(\bmod 4)$, we have $\lambda_{\min }\left(C_{1}^{\prime}\left(\frac{n-2}{2}, \frac{n-2}{2}\right)^{c}\right) \leq \lambda_{\min }\left(C_{1}^{\prime}(p, q)^{c}\right)$ with equality iff $p=\frac{n-2}{2}=q$.

Now, we discuss the classes of graphs having graphs of odd order. 
Lemma 3.7. Suppose that $p \geq 5, q \geq 2$ and $n \geq 13$ are integers with $p, n \equiv 1(\bmod 2)$ and $q \equiv 0(\bmod 2)$. If $p>q+3$, then

$$
\lambda_{\min }\left(C_{2}(p-2, q+2)^{c}\right)<\lambda_{\min }\left(C_{2}(p, q)^{c}\right),
$$

where $p+q+2=n=\left|V\left(C_{2}(p-2, q+2)^{c}\right)\right|=\left|V\left(C_{2}(p, q)^{c}\right)\right|$.

Proof. From equation (2.10), we have $f_{2}(-3, p, q)=117-(p+q)-28 q(p-1)$. Since, for $p \geq 5$ and $q \geq 2$ $f_{2}(-3, p, q)<0$. Therefore, least root of $f_{2}(\lambda, p, q)$ is $\lambda_{2}<-3$. Also, $f_{2}(\lambda, p-2, q+2)=+(5 q-p q)+(-20+$ $4 p-8 q+3 p q) \lambda+(16-8 p-15 q+p q) \lambda^{2}+(39-7 p+11 q-5 p q) \lambda^{3}+(-1+7 p+15 q-2 p q) \lambda^{4}+(-17+6 p+$ $6 q) \lambda^{5}+(-7+p+q) \lambda^{6}-\lambda^{7}$, and

$$
f_{2}(\lambda, p, q)-f_{2}(\lambda, p-2, q+2)=4(\lambda+1)\left(\lambda-\frac{1}{2}\right)\left(\lambda-\frac{\alpha_{1}+\alpha_{2}}{\alpha_{3}}\right)\left(\lambda-\frac{\alpha_{1}-\alpha_{2}}{\alpha_{3}}\right),
$$

where $\alpha_{1}=3-2(p-q), \alpha_{2}=\sqrt{8(p-q)^{2}-32(p-q)+33}$ and $\alpha_{3}=2((p-q)-2)$.

If $p-q \rightarrow 4$, then $\frac{\alpha_{1}+\alpha_{2}}{\alpha_{3}} \rightarrow 0$ and $\frac{\alpha_{1}-\alpha_{2}}{\alpha_{3}} \rightarrow-3$. If $p-q \rightarrow+\infty$, then $\frac{\alpha_{1}+\alpha_{2}}{\alpha_{3}} \rightarrow-1+\sqrt{2}$ and $\frac{\alpha_{1}-\alpha_{2}}{\alpha_{3}} \rightarrow-1-\sqrt{2}$. This shows that for $p-q \geq 4, \frac{\alpha_{1}+\alpha_{2}}{\alpha_{3}} \in\left[0,0.4142\left[\right.\right.$ and $\frac{\alpha_{1}-\alpha_{2}}{\alpha_{3}} \in[-3,-2.4142[$. Thus, for $p>q+3$ and $\lambda<-3$, we have $f_{2}(\lambda, p, q)-f_{2}(\lambda, p-2, q+2)>0$. Also, $f_{2}(-3, p-2, q+2)<0$ which implies that $\lambda_{\text {min }}\left(C_{2}(p-2, q+2)^{c}\right)<\lambda_{\min }\left(C_{2}(p, q)^{c}\right)$.

Lemma 3.8. Suppose that $p \geq 4, q \geq 3$ and $n \geq 13$ are integers with $q, n \equiv 1(\bmod 2)$ and $p \equiv 0(\bmod 2)$. If $p>q+3$, then

$$
\lambda_{\min }\left(C_{2}^{\prime}(p-2, q+2)^{c}\right)<\lambda_{\min }\left(C_{2}^{\prime}(p, q)^{c}\right),
$$

where $p+q+2=n$.

Proof. From equation (2.12), we have $f_{2}^{\prime}(-3, p, q)=117-(p+q)-28 p(q-1)$. Since, for $p \geq 4$ and $q \geq 3$, $f_{2}^{\prime}(-3, p, q)<0$. Therefore, least root of $f_{2}^{\prime}(\lambda, p, q)$ is $\lambda_{2}^{\prime}<-3$. Also,

$$
\begin{gathered}
f_{2}^{\prime}(\lambda, p-2, q+2)=(-4+p+4 q-p q)+(-20+4 p-8 q+3 p q) \lambda+(28-11 p-12 q+p q) \lambda^{2} \\
+(47-9 p+13 q-5 p q) \lambda^{3}+(-1+7 p+15 q-2 p q) \lambda^{4}+(-17+6 p+6 q) \lambda^{5}+(-7+p+q) \lambda^{6}-\lambda^{7},
\end{gathered}
$$

and

$$
f_{2}^{\prime}(\lambda, p, q)-f_{2}^{\prime}(\lambda, p-2, q+2)=2(\lambda+1)(\lambda-0.5)(\lambda-0.1861)(\lambda+2.6861) .
$$

Thus, for $p>q$ and $\lambda<-3$, we have $f_{2}^{\prime}(\lambda, p, q)-f_{2}^{\prime}(\lambda, p-2, q+2)>0$. Also, $f_{2}^{\prime}(-3, p-2, q+2)<0$ which implies that $\lambda_{\text {min }}\left(C_{2}^{\prime}(p-2, q+2)^{c}\right)<\lambda_{\min }\left(C_{2}^{\prime}(p, q)^{c}\right)$.

Corollary 3.9. Suppose that $p \geq 3, q \geq 4$ and $n \geq 13$ are integers with $p, n \equiv 1(\bmod 2)$ and $q \equiv 0(\bmod 2)$. If $q>p+3$, then $\lambda_{\min }\left(C_{2}(p+2, q-2)^{c}\right)<\lambda_{\min }\left(C_{2}(p, q)^{c}\right)$, where $p+q+2=n$.

Proof. Using Lemma 3.8, if $r>s+3$ and $r+s+2=n$ then

$$
\lambda_{\text {min }}\left(C_{2}^{\prime}(r-2, s+2)^{c}\right)<\lambda_{\min }\left(C_{2}^{\prime}(r, s)^{c}\right) .
$$

Therefore, for $n \equiv 1(\bmod 4)$, we have

$$
\begin{aligned}
\lambda_{\min }\left(C_{2}^{\prime}(n-7,5)^{c}\right) & <\lambda_{\min }\left(C_{2}^{\prime}(n-5,3)^{c}\right), \lambda_{\min }\left(C_{2}^{\prime}(n-9,7)^{c}\right) \\
& <\lambda_{\min }\left(C_{2}^{\prime}(n-7,5)^{c}\right), \ldots, \lambda_{\min }\left(C_{2}^{\prime}\left(\frac{n-5}{2}, \frac{n+1}{2}\right)^{c}\right) \\
& <\lambda_{\min }\left(C_{2}^{\prime}\left(\frac{n-1}{2}, \frac{n-3}{2}\right)^{c}\right) .
\end{aligned}
$$

Similarly for $n \equiv 3(\bmod 4)$, we have

$$
\begin{aligned}
\lambda_{\min }\left(C_{2}^{\prime}(n-7,5)^{c}\right) & <\lambda_{\min }\left(C_{2}^{\prime}(n-5,3)^{c}\right), \lambda_{\min }\left(C_{2}^{\prime}(n-9,7)^{c}\right) \\
& <\lambda_{\min }\left(C_{2}^{\prime}(n-7,5)^{c}\right), \ldots, \lambda_{\min }\left(C_{2}^{\prime}\left(\frac{n-3}{2}, \frac{n-1}{2}\right)^{c}\right)
\end{aligned}
$$




$$
<\lambda_{\min }\left(C_{2}^{\prime}\left(\frac{n+1}{2}, \frac{n-5}{2}\right)^{c}\right)
$$

Now, by definition, for $n \equiv 1(\bmod 4), C_{2}^{\prime}(n-5,3) \cong C_{2}(3, n-5), C_{2}^{\prime}(n-7,5) \cong C_{2}(5, n-7), \ldots$, $C_{2}^{\prime}\left(\frac{n-5}{2}, \frac{n+1}{2}\right) \cong C_{2}\left(\frac{n+1}{2}, \frac{n-5}{2}\right)$ and for $n \equiv 3(\bmod 4)^{\prime} C_{2}^{\prime}(n-5,3) \cong C_{2}(3, n-5), C_{2}^{\prime}(n-7,5) \cong C_{2}(5, n-$ $7), \ldots, C_{2}^{\prime}\left(\frac{n-3}{2}, \frac{n-1}{2}\right) \cong C_{2}\left(\frac{n-1}{2}, \frac{n-3}{2}\right)$.

Consequently, $\lambda_{\min }\left(C_{2}(p+2, q-2)^{c}\right)<\lambda_{\min }\left(C_{2}(p, q)^{c}\right)$ for $q>p$, which complete the proof.

Lemma 3.10. Suppose that $p \geq 3$ and $q \geq 2$ are integers with $p \equiv 1(\bmod 2)$ and $q \equiv 0(\bmod 2)$, where $p+q+2=n$ is of the cacti. Then

$$
\lambda_{\text {min }}\left(C_{2}(p, q)^{c}\right) \geq \begin{cases}\lambda_{\text {min }}\left(C_{2}\left(\frac{n+1}{2}, \frac{n-5}{2}\right)^{c}\right) & \text { if } n \equiv 1(\bmod 4) ; \\ \lambda_{\text {min }}\left(C_{2}\left(\frac{n-1}{2}, \frac{n-3}{2}\right)^{c}\right) & \text { if } n \equiv 3(\bmod 4) ;\end{cases}
$$

where equality holds iff $p=\frac{n+1}{2}$ and $q=\frac{n-5}{2}$ with $n \geq 13$, and $p=\frac{n-1}{2}$ and $q=\frac{n-3}{2}$ with $n \geq 15$.

Proof. When $n \equiv 1(\bmod 4)$, then for $p=\frac{n+1}{2}$ and $q=\frac{n-5}{2}$, the equations (2.10) becomes $f_{2}\left(-3, \frac{n+1}{2}, \frac{n-5}{2}\right)=$ $-(n-7.4647)(n+1.6075)$. Thus, for $n \geq 13$, we have $f_{2}\left(-3, \frac{n+1}{2}, \frac{n-5}{2}\right)<0$. (b) When $n \equiv 3(\bmod 4)$, then for $p=\frac{n-1}{2}$ and $q=\frac{n-3}{2}$, the equation (2.10) becomes, $f_{2}\left(-3, \frac{n-1}{2}, \frac{n-3}{2}\right)=-(n-5.7569)(n-0.1001)$. So, for $n \geq 15$, we have $f_{2}\left(-3, \frac{n-1}{2}, \frac{n-3}{2}\right)<0$. Thus, from both the cases least root of $f_{2}(\lambda, p, q)$ is $\lambda_{2}<-3$.

Now, by Lemma 3.7, if $p$ is greater than $q+3$ and $\lambda$ is less than -3 , then $\lambda_{\min }\left(C_{2}(p-2, q+2)^{c}\right)<$ $\lambda_{\min }\left(C_{2}(p, q)^{c}\right)$ and by Corollary 3.9, if $q>p, q-p \neq 2$ and $\lambda<-3$, then $\lambda_{\text {min }}\left(C_{2}(p+2, q-2)^{c}\right)<\lambda_{\text {min }}\left(C_{2}(p, q)^{c}\right)$.

Consequently, for $n \geq 13$ and $n \equiv 1(\bmod 4)$, we have $\lambda_{\min }\left(C_{2}\left(\frac{n+1}{2}, \frac{n-5}{2}\right)^{c}\right) \leq \lambda_{\min }\left(C_{2}(p, q)^{c}\right)$ with equality if $p=\frac{n+1}{2}$ and $p=\frac{n-5}{2}$, and (b) for $n \geq 15$ and $n \equiv 3(\bmod 4)$, we have $\lambda_{\min }\left(C_{2}\left(\frac{n-1}{2}, \frac{n-3}{2}\right)^{c}\right) \leq \lambda_{\min }\left(C_{2}(p, q)^{c}\right)$ with equality iff $p=\frac{n-1}{2}$ and $q=\frac{n-3}{2}$. This complete the proof.

\section{Characterization}

This section includes the main results in which minimizing graphs are characterized in the family of connected graphs with the condition that the complement of each graph is a cactus such that either its each block is only an edge or it has at least one block which is an edge and at least one block which is a cycle. In Lemma 4.1 and 4.2, the basic results are developed which are used in the main results. In Lemma 4.3 and Lemma 4.4, minimizing graphs are characterized in $\Omega_{1, n}^{c}$ and $\Omega_{2, n}^{c}$ respectively. Finally, in Theorem 4.5 minimizing graphs are characterized in $\Omega_{n}^{c}=\Omega_{1, n}^{c} \cup \Omega_{2, n}^{c}$.

Lemma 4.1. Let $n \geq 12$ and $X=\left(X_{1}, X_{2}, X_{3}, \ldots, X_{n}\right)^{T}$ be a real vector defined on $C \in \Omega_{n}$ such that $\left|X_{1}\right| \geq\left|X_{2}\right| \geq$ $\left|X_{3}\right| \geq \ldots \geq\left|X_{n}\right|$ and all $X_{i}$ are either non-negative or non-positive. Then

$$
\sum_{u v \in E(C)} X_{u} X_{v} \leq\left\{\begin{array}{cl}
\sum_{u v \in E\left(B_{1}(n-1)\right)} X_{u} X_{v} & \text { if } n \equiv 1(\bmod 2) ; \\
\sum_{u v \in E\left(B_{2}(n-1)\right)} X_{u} X_{v} & \text { if } n \equiv 0(\bmod 2) ;
\end{array}\right.
$$

where equality holds if $C \cong B_{1}(n-1)$ and $C \cong B_{2}(n-1)$ respectively.

Proof. Suppose that $X$ is non-negative and discuss the following two cases:

Case-1. Suppose that $C \in \Omega_{n}$ is a cactus graph such that its each block is an edge i.e. $C \in \Omega_{1, n}$. Let $X_{1}$ be the value of the vertex $v \in C$ assigned by $X$. As $n \geq 10$ and is not a bundle, therefore there exist a vertex in $C$ say $u$ which is not adjacent to $v$. Thus, a vertex $w$ adjacent to $u$ ( $w \sim u$ ) exists on a path from $v$ to $u$ as $C$ is connected. A new cactus $\tilde{C}$ having each block as an edge can be found on the deletion of the edge $w u$ and addition of $v u$ in $C$. We find a star $K_{1, n-1}$ with center $v$ by repeating the same process for the non-neighbor of $v$ in the cactus $\tilde{C}$ and so on. Thus, we have

$$
\sum_{u v \in E(C)} X_{u} X_{v} \leq \sum_{u v \in E(\tilde{C})} X_{u} X_{v} \leq \sum_{i=2}^{n} X_{1} X_{i}=\sum_{u v \in E\left(K_{1, n-1}\right)} X_{u} X_{v}
$$


Case-2. Suppose that $C \in \Omega_{n}$ is a cactus graph such that it has at least one block which is an edge and at least one block which is a cycle i.e. $C \in \Omega_{2, n}$. Assume that $k$ is number of cycles in $C$, then $1 \leq k \leq\left\lceil\frac{n-2}{2}\right\rceil$, where $n$ is order of $C$. Now, we delete an edge in each cycle under the conditions, (i) no two deleted edges of any two cycles have common vertex and (ii) the deleted edge in a cycle which has the vertex with label $X_{1}$ is not incident on this vertex. Thus, we have a cactus graph such that its each block is an edge, say $C^{\prime}$ i.e. $C^{\prime} \in \Omega_{1, n}$. Then by Case 1, we have $\sum_{u v \in E\left(C^{\prime}\right)} X_{u} X_{v} \leq \sum_{u v \in E\left(K_{1, n-1}\right)} X_{u} X_{v}$. Now, we add the deleted edges, then

$$
\begin{aligned}
\sum_{u v \in E(C)} X_{u} X_{v} & =\sum_{u v \in E\left(C^{\prime}\right)} X_{u} X_{v}+\underbrace{\left(X_{v_{r_{1}}} X_{v_{r_{1}+1}}+X_{v_{r_{2}}} X_{v_{r_{2}+1}+, \ldots,+}+X_{v_{r_{k}}} X_{v_{r_{k}+1}}\right)}_{k \text { terms }} \\
& \leq \sum_{u v \in E\left(K_{1, n-1}\right)} X_{u} X_{v}+\left(X_{v_{r_{1}}} X_{v_{r_{1}+1}}+X_{v_{r_{2}}} X_{v_{r_{2}+1}}+\ldots,+X_{v_{r_{k}}} X_{v_{r_{k}+1}}\right),
\end{aligned}
$$

where $v_{r}, v_{r+1}, v_{s}, v_{s+1}, \ldots, v_{t}, v_{t+1}$ are $2 k$ distinct vertices of $C$. Moreover, the inequality in (4.1) does not disturb, if we add $k$ terms $\left(X_{v_{r_{1}}} X_{v_{r_{1}+1}}+X_{v_{r_{2}}} X_{v_{r_{2}+1}}+\ldots,+X_{v_{r_{k}}} X_{v_{r_{k}+1}}\right)$ in its right hand side. Consequently, from both the cases, we have

$$
\sum_{u v \in E(C)} X_{u} X_{v} \leq \sum_{u v \in E\left(K_{1, n-1}\right)} X_{u} X_{v}+\left(X_{v_{r_{1}}} X_{v_{r_{1}+1}}+X_{v_{r_{2}}} X_{v_{r_{2}+1}}+, \ldots,+X_{v_{r_{k}}} X_{v_{r_{k}+1}}\right) .
$$

(a) Assume $n \equiv 1(\bmod 2)$. Since, in $K_{1, n-1}$, one vertex, say $v_{1}$ with $d\left(v_{1}\right)=n-1$ has value $X_{1}$ and remaining $n-1$ pendent vertices have values $X_{i}$ for $2 \leq i \leq n$. Pairing the vertices of degree 1 and joining each pair by an edge, we have $\frac{n-1}{2}$ edges $v_{i} v_{i+1}$, where $i \in I=\{2,4, \ldots, n-1\}$. Thus, $\left(X_{v_{r}} X_{v_{r+1}}+X_{v_{s}} X_{v_{s+1}}+, \ldots,+X_{v_{t}} X_{v_{t+1}}\right) \leq$ $\sum_{i \in I} X_{i} X_{i+1}$. Since, $\sum_{u v \in E\left(K_{1, n-1}\right)} X_{u} X_{v}+\sum_{i \in I} X_{i} X_{i+1}=\sum_{u v \in E\left(B_{1}(n-1)\right)} X_{u} X_{v}$. Consequently (4.2) becomes, $\sum_{u v \in E(C)} X_{u} X_{v} \leq$ $\sum_{u v \in E\left(B_{1}(n-1)\right)} X_{u} X_{v}$, where equality holds if $C$ has one vertex of degree $n-1$ and remaining $n-1$ vertices are of degree two, i.e. $C \cong B_{1}(n-1)$.

(b) Assume $n \equiv 0(\bmod 2)$. Since, in $K_{1, n-1}$, one vertex, say $v_{1}$ with $d\left(v_{1}\right)=n-1$ has value $X_{1}$ and remaining $n-1$ pendent vertices have values $X_{i}$ for $2 \leq i \leq n$. Pairing the $n-2$ vertices of degree 1 and joining each pair by an edge, we have $\frac{n-2}{2}$ edges $v_{i} v_{i+1}$, where $i \in I=\{2,4, \ldots, n-2\}$. Thus, $\left(X_{v_{r_{1}}} X_{v_{r_{1}+1}}+X_{v_{r_{2}}} X_{v_{r_{2}+1}}+, \ldots,+X_{v_{r_{k}}} X_{v_{r_{k}+1}}\right) \leq \sum_{i \in I} X_{i} X_{i+1}$. Since, $\sum_{u v \in E\left(K_{1, n-1}\right)} X_{u} X_{v}+\sum_{i \in I} X_{i} X_{i+1}=\sum_{u v \in E\left(B_{2}(n-1)\right)} X_{u} X_{v}$. So, (4.2) takes form $\sum_{u v \in E(C)} X_{u} X_{v} \leq \sum_{u v \in E\left(B_{2}(n-1)\right)} X_{u} X_{v}$, where equality holds if $C$ has one pendent vertex, one of degree $n-1$ and $n-2$ with degree 2, i.e. $C=B_{2}(n-1)$.

Lemma 4.2. For $n \geq 12$ and $C^{c} \in \Omega_{n}^{c}$, the first eigenvector $X$ of $C^{c}$ has at least 2 negative and 2 positive entries.

Proof. Assume that there is a unique vertex $v \in C^{c}$ having positive value labeled by $X$. The degree of $v$ in $C^{c}$ is non-zero, i.e $d_{C^{c}}(v) \neq 0$. As, if $d_{C^{c}}(v)=0$, then $C$ is a bundle, which is a contradiction to the construction of $\Omega_{n}^{c}$. Consequently, $1 \leq d_{C^{c}}(v) \leq n-1$. Let $u$ be another vertex in $C^{c}$. Since all the vertices are supposed to have negative values except $v$, we claim $u \sim v$, otherwise (2.2) does not holds for $u \in C^{c}$ as $\lambda X_{u}>0$ and $\sum_{w \in N_{G}(u)} X_{w}<0 \Rightarrow \lambda X_{u} \neq \sum_{w \in N_{G}(u)} X_{w}$. Consequently, $d_{C^{c}}(v)=n-1$ for each $v \in C^{c}$. It shows that $C$ is disconnected, which is a contradiction to the construction of $\Omega_{n}^{c}$. Similarly, we can prove, if $v \in C^{c}$ is a unique vertex with negative value assigned by $X$.

Lemma 4.3. Suppose that $C$ is a cactus graph such that $C^{c} \in \Omega_{2, n}^{c}$ and $|V(C)|=n=p+q+2 \geq 12$.

(a) If $n \equiv 0(\bmod 2)$, then either $\lambda_{\min }\left(C_{1}(p, q)^{c}\right) \leq \lambda_{\min }\left(C^{c}\right)$, or $\lambda_{\min }\left(C_{1}^{\prime}(p, q)^{c}\right) \leq \lambda_{\min }\left(C^{c}\right)$, where equalities hold if $C \cong C_{1}(p, q)$ or $C \cong C_{1}^{\prime}(p, q)$, respectively.

(b) If $n \equiv 1(\bmod 2)$, then $\lambda_{\min }\left(C_{2}(p, q)^{c}\right) \leq \lambda_{\min }\left(C^{c}\right)$ with equality if $C \cong C_{2}(p, q)$.

Proof. Assume $X$ is a unit first eigenvector of $C^{c}$. Define $V_{+}=\left\{v: X_{v} \geq 0, v \in V\left(C^{c}\right)\right\}$ and $V_{-}=\left\{v: X_{v}<\right.$ $\left.0, v \in V\left(C^{c}\right)\right\}$ such that $\left|V_{+}\right|,\left|V_{-}\right| \geq 2$ by Lemma 4.2. Suppose that the subgraphs $C_{+}$and $C_{-}$of $C$ are induced 
by the vertex sets $V_{+}$and $V_{-}$respectively and $E^{\prime} \neq \Phi$ is subset of $E(C)$ with one end in $C_{+}$and other in $C_{-}$. Thus, we have

$$
\sum_{u v \in E(C)} X_{u} X_{v}=\sum_{u v \in E\left(C_{+}\right)} X_{u} X_{v}+\sum_{u v \in E\left(C_{-}\right)} X_{u} X_{v}+\sum_{u v \in E^{\prime}} X_{u} X_{v}
$$

(a) Assume that $n \equiv 0(\bmod 2)$, where $n=p+q+2$. Let $\bar{C}$ be a graph obtained from $C$ by some possible addition or deletion of edges in $C_{+}$and $C_{-}$such that the subgraph $\bar{C}_{+}$and $\bar{C}_{-}$of $\bar{C}$ induced by $C_{+}$and $C_{-}$are cactus graphs satisfying one of the following possibilities, (i) each block of one of the subgraphs $\bar{C}_{+}$and $\bar{C}_{-}$ is an edge and other has at least one block which is a cycle and at least one block which is an edge, (ii) all the blocks of both the subgraphs $\bar{C}_{+}$and $\bar{C}_{-}$are cycles, (iii) all the blocks of one subgraph are edges and of other are cycles, (iv) each block of one of the subgraphs $\bar{C}_{+}$and $\bar{C}_{-}$is a cycle and other has at least one block which is a cycle and at least one block which is an edge, and (v) both the subgraphs $\bar{C}_{+}$and $\bar{C}_{-}$have at least one block which is a cycle and at least one block which is an edge.

For (i), suppose $\bar{C}_{+}$is a cactus such that its each block is an edge, otherwise we take $-X$ as a first eigenvector. Let $u$ ' be a vertex of $\bar{C}_{+}$with maximum modulus among all the vertices, then by discussion of equation (4.1) in Lemma 4.1, we obtain a cactus with each block as an edge which is infect a star $K_{1, p}$. Similarly, suppose that $v^{\prime}$ is a vertex with maximum modulus among all the vertices of $\bar{C}_{-}$. Firstly, we delete an edge in each block such that no two deleted edges of any two blocks have a common vertex in $\bar{C}_{\text {- }}$ and the deleted edge in a block which has $v^{\prime}$ is not incident on this vertex. Thus, we obtain a subgraph of $\bar{C}_{\text {- such }}$ that its each block is an edge. Then by the same discussion as of equation (4.2) in Lemma 4.1, we obtain a cactus with at least one block as a cycle and at least one block as an edge which is infect a star $K_{1, q}$ with edges among the pendent vertices having different end points.

Since $n \equiv 0(\bmod 2)$ and $n=p+q+2$, where $n=\left|V_{+} \cup V_{-}\right|=\left|\bar{C}_{+} \cup \bar{C}_{-}\right|, p+1=\left|V_{+}\right|=\left|\bar{C}_{+}\right|$and $q+1=\left|V_{-}\right|=\left|\bar{C}_{-}\right|$. Therefore, either both $p$ and $q$ are even or odd.

Suppose $p$ and $q$ both are even. By pairing the pendent vertices of the star $K_{1, p}$ which is obtained from $\bar{C}_{+}$ and joining them by edges, we have a bundle $B_{1}(p)$ with center $u$ ' having maximum modulus value, where $p+1=\left|V_{+}\right| \geq 3$ is odd. Similarly, pair the remaining possible pendent vertices of the subgraph obtained from $\bar{C}_{-}$and join them by edges. Thus, we obtain a bundle $B_{1}(q)$ with center $v^{\prime}$ having maximum modulus value, where $q+1=\left|V_{+}\right| \geq 3$ is odd. Thus, by Lemma 4.1 (a), we have

$$
\sum_{u v \in E\left(C_{+}\right)} X_{u} X_{v} \leq \sum_{u v \in E\left(\bar{C}_{+}\right)} X_{u} X_{v} \leq \sum_{u v \in E\left(B_{1}(p)\right)} X_{u} X_{v}
$$

and

$$
\sum_{u v \in E\left(C_{-}\right)} X_{u} X_{v} \leq \sum_{u v \in E\left(\bar{C}_{-}\right)} X_{u} X_{v} \leq \sum_{u v \in E\left(B_{1}(q)\right)} X_{u} X_{v} .
$$

Suppose $p$ and $q$ both are odd. By pairing the pendent vertices of the star which is obtained from $\bar{C}_{+}$ and joining them by edges, we have a bundle $B_{2}(p)$ with center $u$ ' having maximum modulus value, where $p+1=\left|V_{+}\right| \geq 4$ is even. Similarly, pair the remaining possible pendent vertices of the subgraph obtained from $\bar{C}_{-}$and join them by edges. Thus, we obtain a bundle $B_{2}(q)$ with center $v^{\prime}$ having maximum modulus value, where $q+1=\left|V_{+}\right| \geq 4$ is even. Thus, by Lemma 4.1 (b), we have

$$
\begin{gathered}
\sum_{u v \in E\left(C_{+}\right)} X_{u} X_{v} \leq \sum_{u v \in E\left(\bar{C}_{+}\right)} X_{u} X_{v} \leq \sum_{u v \in E\left(B_{2}(p)\right)} X_{u} X_{v}, \\
\sum_{u v \in E\left(C_{-}\right)} X_{u} X_{v} \leq \sum_{u v \in E\left(\bar{C}_{-}\right)} X_{u} X_{v} \leq \sum_{u v \in E\left(B_{2}(q)\right)} X_{u} X_{v} .
\end{gathered}
$$

Assume that $u^{\prime \prime} \in \bar{C}_{+}$and $v^{\prime \prime} \in \bar{C}_{-}$have minimum modulus values. Then

$$
\sum_{u v \in E^{\prime}} X_{u} X_{v} \leq X_{u^{\prime \prime}} X_{v^{\prime \prime}}
$$


Using (4.4), (4.5), (4.8) and (4.6), (4.7), (4.8) in (4.3) respectively, we have

$$
\begin{gathered}
\sum_{u v \in E(C)} X_{u} X_{v} \leq \sum_{u v \in E\left(B_{1}(p)\right)} X_{u} X_{v}+\sum_{u v \in E\left(B_{1}(q)\right)} X_{u} X_{v}+X_{u^{\prime \prime}} X_{v^{\prime \prime}}, \\
\sum_{u v \in E(C)} X_{u} X_{v} \leq \sum_{u v \in E\left(B_{2}(p)\right)} X_{u} X_{v}+\sum_{u v \in E\left(B_{2}(q)\right)} X_{u} X_{v}+X_{u^{\prime \prime}} X_{v^{\prime \prime}} .
\end{gathered}
$$

Since $p \geq q \geq 2$. Therefore, if we take $u^{\prime \prime} \in B_{1}(p), v^{\prime \prime} \in B_{1}(q)$ of degree 2 and $u^{\prime \prime} \in B_{2}(p), v^{\prime \prime} \in B_{2}(q)$ of degree 1. Then (4.9) and (4.10) becomes,

$$
\begin{gathered}
\sum_{u v \in E(C)} X_{u} X_{v} \leq \sum_{u v \in E\left(C_{1}(p, q)\right)} X_{u} X_{v}, \\
\sum_{u v \in E(C)} X_{u} X_{v} \leq \sum_{u v \in E\left(C_{1}^{\prime}(p, q)\right)} X_{u} X_{v} .
\end{gathered}
$$

Now by the equations (2.1)-(2.4) and (4.11), we have $\lambda_{\min }\left(C^{c}\right)=X^{T} A\left(C^{c}\right) X=X^{T}(J-I-A(C)) X=X^{T}(J-I) X-$ $X^{T} A(C) X \geq X^{T}(J-I) X-X^{T} A\left(C_{1}(p, q)\right) X=X^{T} A\left(C_{1}(p, q)^{c}\right) X \geq \lambda_{\text {min }}\left(C_{1}(p, q)^{c}\right) \Rightarrow \lambda_{\text {min }}\left(C_{1}(p, q)^{c}\right) \leq \lambda_{\text {min }}\left(C^{c}\right)$. Similarly by equations (2.1)-(2.4) and (4.12), we have $\lambda_{\min }\left(C_{1}^{\prime}(p, q)^{c}\right) \leq \lambda_{\min }\left(C^{C}\right)$.

Thus, for $n \equiv 0(\bmod 2)$, either $\lambda_{\min }\left(C_{1}(p, q)^{c}\right) \leq \lambda_{\min }\left(C^{c}\right)$ or $\lambda_{\min }\left(C_{1}^{\prime}(p, q)^{c}\right) \leq \lambda_{\min }\left(C^{c}\right)$, where $p+q+2=$ $n$. On the same way, it can be prove that the results are also true for all the possibilities (ii)-(v).

(b) Assume that $n \equiv 1(\bmod 2)$, where $n=p+q+2$. Let $\bar{C}$ be a graph obtained from $C$ with subgraph $\bar{C}_{+}$ and $\bar{C}_{-}$induced from $C_{+}$and $C_{-}$are cactus graphs satisfying any one of the possibilities which are stated in (a). For (i), we proceed same as in (a) and find the cactus graphs which are infect stars with some possible edges among the pendent vertices having different end points from both the subgraphs $\bar{C}_{+}$and $\bar{C}_{-}$after the deletion and addition of some edges. Since $n \equiv 1(\bmod 2)$ and $n=p+q+2$, where $n=\left|V_{+} \cup V_{-}\right|=\left|\bar{C}_{+} \cup \bar{C}_{-}\right|$, $p+1=\left|V_{+}\right|=\left|\bar{C}_{+}\right|$and $q+1=\left|V_{-}\right|=\left|\bar{C}_{-}\right|$. Therefore, either $p$ is even and $q$ is odd or vice versa. Without loss of generality, we assume $p$ as odd and $q$ even.

Suppose that $u^{\prime}$ and $u^{\prime \prime}$ in $\bar{C}_{+}$, and $v^{\prime}$ and $v^{\prime \prime}$ in $\bar{C}_{-}$have have maximum and minimum modulus values respectively. Then by the same discussion as in (a) with the help of Lemma 4.1, we have

$$
\begin{gathered}
\sum_{u v \in E\left(C_{+}\right)} X_{u} X_{v} \leq \sum_{u v \in E\left(\bar{C}_{+}\right)} X_{u} X_{v} \leq \sum_{u v \in E\left(B_{2}(p)\right)} X_{u} X_{v}, \\
\sum_{u v \in E\left(C_{-}\right)} X_{u} X_{v} \leq \sum_{u v \in E\left(\bar{C}_{-}\right)} X_{u} X_{v} \leq \sum_{u v \in E\left(B_{1}(q)\right)} X_{u} X_{v},
\end{gathered}
$$

and

$$
\sum_{u v \in E^{\prime}} X_{u} X_{v} \leq X_{u^{\prime \prime}} X_{v^{\prime \prime}}
$$

where the bundle $B_{2}(p)$ has center $u^{\prime}$ with maximum modulus value and $p+1=\left|V_{+}\right| \geq 4$ is even. Similarly, the bundle $B_{1}(q)$ has center $v^{\prime}$, with maximum modulus value and $q+1=\left|V_{+}\right| \geq 3$ is odd. Now, using (4.13), (4.14) and (4.15) in (4.3), we have

$$
\sum_{u v \in E(C)} X_{u} X_{v} \leq \sum_{u v \in E\left(B_{2}(p)\right)} X_{u} X_{v}+\sum_{u v \in E\left(B_{1}(q)\right)} X_{u} X_{v}+X_{u^{\prime \prime}} X_{v^{\prime \prime}}
$$

Since $p, q \geq 2$. Therefore, if we take $u^{\prime \prime} \in B_{2}(p)$ and $v^{\prime \prime} \in B_{1}(p)$ such that degree of $u^{\prime \prime}$ in $B_{2}(p)$ is 1 and degree of $v^{\prime \prime}$ in $B_{1}(q)$ is 2 . Thus, (4.16) becomes

$$
\sum_{u v \in E(C)} X_{u} X_{v} \leq \sum_{u v \in E\left(C_{2}(p, q)\right)} X_{u} X_{v}
$$


Now by the equations (2.1)-(2.4) and (4.17), $\lambda_{\min }\left(C_{2}(p, q)^{c}\right) \leq \lambda_{\min }\left(C^{c}\right)$, where $n=p+q+2>10$ and $n \equiv 1(\bmod 2)$. Similarly, it also can be prove for all other possibilities.

Now finally we prove that there does not exist any vertex in $V_{+}$such that its value given by $X$ is zero and $E^{\prime}$ has exactly one edge. Firstly, among the vertices of $C_{1}(p, q)$, we prove that $v_{2}=u^{\prime}$ and $v_{4}=u^{\prime \prime}$ are unique ones in $C_{+}$and, $v_{5}=v^{\prime \prime}$ and $v_{7}=v^{\prime}$ are unique ones in $C_{-}$with maximum and minimum modulus, respectively. For this, we will show $0 \leq X_{4}<X_{3}<X_{1}<X_{2}$ and $X_{7}<X_{8}<X_{6}<X_{5}<0$. By Lemma 4.2, we have $X_{1}, X_{2}, X_{3}$ non negative and $X_{4}, X_{5}, X_{6}, X_{7}, X_{8}$ negative values in the first eigenvector $X$ of $C_{1}(p, q)^{c}$. By (2.5), $\lambda_{1}\left(X_{2}-X_{1}\right)=-(p-4) X_{1}-X_{3}-X_{4}<0,\left(\lambda_{1}+1\right)\left(X_{1}-X_{3}\right)=-\left(X_{1}-X_{4}\right)<0$, and $\lambda_{1}\left(X_{3}-X_{4}\right)=X_{5}<0 \Rightarrow$ $X_{2}-X_{1}>0, X_{1}-X_{3}>0$ and $X_{3}-X_{4}>0$. Thus

$$
0 \leq X_{4}<X_{3}<X_{1}<X_{2}
$$

Similarly, $\lambda_{1}\left(X_{8}-X_{7}\right)=X_{5}+X_{6}+(q-4) X_{8}<0,\left(\lambda_{1}+1\right)\left(X_{6}-X_{8}\right)=-X_{5}+X_{8}<0$ and $\lambda_{1}\left(X_{5}-X_{6}\right)=-X_{4}<0$ $\Rightarrow X_{8}-X_{7}>0, X_{5}-X_{6}>0$ and $X_{6}-X_{8}>0$. Thus

$$
X_{7}<X_{8}<X_{6}<X_{5}<0
$$

If any one of the vertices $v_{1}, v_{2}$ and $v_{3}$ has value zero assigned by $X$, then by (4.18) $X_{3}=0=X_{4}$. Moreover, by (2.5), we have $X_{5}=0=X_{6}$, which is a contradiction to the construction of $V_{-}$and $C_{-}$. If the value of the vertex $v_{4}$ labeled by $X$ is zero, then by (2.5), $\lambda_{1}\left(X_{5}-X_{6}\right)=0 \Rightarrow X_{5}=X_{6}$ which is a contradiction to (4.19) (i.e. $X_{5}$ is a unique one in $C_{-}$). Consequently, $X_{1}, X_{2}, X_{3}$ and $X_{4}$ are non zero positive values of $X$. Thus, $v \notin V_{+}$such that $X_{V}=0$. By (4.4), (4.5), (4.8), (4.9) and the above discussion, we have $C_{+}=\bar{C}_{+}=B_{1}(p), C_{-}=\bar{C}_{-}=B_{1}(q)$ and $E^{\prime}$ has only one edge $u^{\prime \prime} v^{\prime \prime}=v_{4} v_{5}$ in $B_{1}(p, q)$. Similarly, we can prove for $B_{1}^{\prime}(p, q)$ and $B_{2}(p, q)$. This complete the proof.

Similarly, we can prove the following result:

Lemma 4.4. Suppose that $C$ is a cactus graph of order $n=p+q+2 \geq 10$ such that $C^{c} \in \Omega_{1, n}^{c}$.

(a) If $n \equiv 0(\bmod 2)$, then either $\lambda_{\text {min }}\left(C_{1}(p, q)^{c}\right)<\lambda_{\text {min }}\left(C^{c}\right)$ or $\lambda_{\text {min }}\left(C_{1}^{\prime}(p, q)^{c}\right)<\lambda_{\text {min }}\left(C^{c}\right)$.

(b) If $n \equiv 1(\bmod 2)$, then $\lambda_{\min }\left(C_{2}(p, q)^{c}\right)<\lambda_{\min }\left(C^{c}\right)$.

Theorem 4.5. Suppose that $C$ is a cactus graph of order $n$ such that $C^{c} \in \Omega_{n}^{c}=\Omega_{1, n}^{c} \cup \Omega_{2, n}^{c}$.

(1)Assume that $n \equiv 0(\bmod 2), p, q \geq 4$ and $n=p+q+2$ :

(a)For $p, q \equiv 0(\bmod 2)$;

(i)If $n \geq 14$ and $n \equiv 2(\bmod 4)$, then $\lambda_{\min }\left(C_{1}\left(\frac{n-2}{2}, \frac{n-2}{2}\right)^{c}\right) \leq \lambda_{\min }\left(C_{1}(p, q)^{c}\right) \leq \lambda_{\min }\left(C^{c}\right)$, where equalities hold if $C \cong C_{1}\left(\frac{n-2}{2}, \frac{n-2}{2}\right) \cong \lambda_{\min }\left(C_{1}(p, q)\right)$,

(ii)If $n \geq 12$ and $n \equiv 0(\bmod 4)$, then $\lambda_{\min }\left(C_{1}\left(\frac{n}{2}, \frac{n-4}{2}\right)^{c}\right) \leq \lambda_{\min }\left(C_{1}(p, q)^{c}\right) \leq \lambda_{\min }\left(C^{c}\right)$, where equalities hold if $C \cong C_{1}\left(\frac{n}{2}, \frac{n-4}{2}\right) \cong \lambda_{\min }\left(C_{1}(p, q)\right)$,

(b)For $p, q \equiv 1(\bmod 2)$;

(i)If $n \geq 14$ and $n \equiv 2(\bmod 4)$, then $\lambda_{\min }\left(C_{1}^{\prime}\left(\frac{n}{2}, \frac{n-4}{2}\right)^{c}\right) \leq \lambda_{\min }\left(C_{1}^{\prime}(p, q)^{c}\right) \leq \lambda_{\min }\left(C^{c}\right)$, where equalities hold if $C \cong C_{1}^{\prime}\left(\frac{n}{2}, \frac{n-4}{2}\right) \cong \lambda_{\min }\left(C_{1}^{\prime}(p, q)\right)$,

(ii)If $n \geq 12$ and $n \equiv 0(\bmod 4)$, then $\lambda_{\min }\left(C_{1}^{\prime}\left(\frac{n-2}{2}, \frac{n-2}{2}\right)^{c}\right) \leq \lambda_{\min }\left(C_{1}^{\prime}(p, q)^{c}\right) \leq \lambda_{\min }\left(C^{c}\right)$, where equalities hold if $C \cong C_{1}^{\prime}\left(\frac{n-2}{2}, \frac{n-2}{2}\right) \cong \lambda_{\min }\left(C_{1}^{\prime}(p, q)\right)$.

(2) Assume that $n \equiv 1(\bmod 2), p \equiv 1(\bmod 2)$ and $q \equiv 0(\bmod 2)$ :

(i)If $n \geq 13$ and $n \equiv 1(\bmod 4)$, then $\lambda_{\min }\left(C_{2}\left(\frac{n+1}{2}, \frac{n-5}{2}\right)^{c}\right) \leq \lambda_{\min }\left(C_{2}(p, q)^{c}\right) \leq \lambda_{\min }\left(C^{c}\right)$, where equalities hold if $C \cong C_{2}\left(\frac{n+1}{2}, \frac{n-5}{2}\right) \cong \lambda_{\min }\left(C_{2}(p, q)\right)$,

(ii)If $n \geq 15$ and $n \equiv 3(\bmod 4)$, then $\lambda_{\min }\left(C_{2}\left(\frac{n-1}{2}, \frac{n-3}{2}\right)^{c}\right) \leq \lambda_{\min }\left(C_{2}(p, q)^{c}\right) \leq \lambda_{\min }\left(C^{c}\right)$, with equality if $C \cong C_{2}\left(\frac{n-1}{2}, \frac{n-3}{2}\right) \cong \lambda_{\text {min }}\left(C_{2}(p, q)\right)$.

Proof. The result follows from Lemma 3.3-Lemma 3.5 and Lemma 4.3-Lemma 4.4. 


\section{Conclusions}

Petrovic et al. [23] explored a unique cactus as a minimizing graph from the class of cacti such that the order of each cactus is $n$. But, it is noted that the complement of the proposed minimizing graph is disconnected. In this paper, we characterize the minimizing graphs in a collection of connected graphs such that the complement of each graph of order $n$ is a cactus with the condition that either its each block is only an edge or it has at least one block which is an edge and at least one block which is a cycle. However, the problem is still open to characterize the minimizing graphs in a collection of connected graphs whose complements are in the complete class of cacti (each block of a cactus is only an edge, at least one block is an edge and at least one block is a cycle, or each block is a cycle).

Acknowledgement: The authors are indebted to the anonymous referee for his valuable comments to improve the original version of this paper. The first author is supported by NSFC of China (No.11701530) and the Fundamental Research Funds for the Central Universities (No.2652017146).

\section{References}

[1] Collatz L., Sinogowitz U., Spektren endlicher Grafen, Abhandlungen aus dem Mathematischen Seminar der Universität Hamburg, 1957, 21, 63-77.

[2] Cvetković D., Rowlinson P., The largest eigenvalues of a graph: a survey, Linear Multileaner Algebra, 1990, 28, 3-33.

[3] Cvetković D., Doob M., Sachs H., Spectra of Graphs, 3rd ed. Johann Ambrosius Barth, Heidelberg, 1995.

[4] Cretković D., Rowlinson P., Simić S., Spectral Generalizations of Line Graphs: on Graph with Least Eigenvalue -2, Cambridge Uni. Press, London Math. Soc., 2014.

[5] Hong Y., Shu J., Sharp lower bounds of the least eigenvalue of planar graphs, Linear Algebra Appl., 1999, 296, $227-232$.

[6] Bell F.K., Cvetković D., Rowlinson P., Simić S., Graph for which the least eigenvalues is minimal, II, Linear Algebra Appl., 2008, 429, 2168-2179.

[7] Du Z., Spectral properties of a class of unicyclic graphs, J. Inequal. Appl., 2017, Article number: 96

[8] Guan M., Zhai M., Wu Y., On the sum of two largest Laplacian eigenvalue of trees, J. Inequal. Appl., 2014, Article number: 242.

[9] Guo S.-G., Liu X., Zhang R., Yu G., Ordering non-bipartite unicyclic graphs with pendant vertices by the least Q-eigenvalue, J. Inequal. Appl., 2016, 2016: 136.

[10] Petrović M., Borovićanin B., Aleksić T., Bicyclic graphs for which the least eigenvalue is minimum, Linear Algebra Appl., 2009, 430, 1328-1335.

[11] Petrović M., Aleksić T., Simić S., Further results on the least eigenvalue of connected graphs, Linear Algebra Appl., 2011, 435, 2303-2313.

[12] Zheng Y., Chang A., Li J., Rula S., Bicyclic graphs with maximum sum of the two largest Laplacian eigenvalues, J. Inequal. Appl., 2016, Article number: 287.

[13] Zheng Y., Chang A., Li J., On the sum of two largest Laplacian eigenvalue of unicyclic graphs, J. Inequal. Appl., 2015, Article number: 275.

[14] Bell F.K., Cvetković D., Rowlinson P., Simić S., Graph for which the least eigenvalues is minimal, I, Linear Algebra Appl., 2008, 429, 234-241.

[15] Fan Y.-Z., Zhang F.-F., Wang Y., The least eigenvalue of the complements of trees, Linear Algebra Appl., 2011, 435, $2150-2155$.

[16] Javaid M., Minimizing graph of the connected graphs whose complements are bicyclic with two cycles, Turk. J. Math., 2017, 41, 1433-1445.

[17] Javaid M., Characterization of the minimizing graph of the connected graphs whose complements are bicyclic, Mathematics, 2017, 5, 18, doi:10.3390/math5010018.

[18] Wang Y., Fan Y.-Z., Li X.-X., Zhang F.-F., The least eigenvalue of graphs whose complements are unicyclic, Discuss. Math. Graph Theory, 2015, 35(2), 249-260.

[19] Javaid M., On the second minimizing graph in the set of complements of trees, AKCE Int. J. Graphs Comb., 2018, https://doi.org/10.1016/j.akcej.2018.11.005.

[20] Sudhakar S., Francis S., Balaji V., Odd mean labeling for two star graph, Appl. Math. Nonlinear Sci., 2017, 2(1), $195-200$.

[21] Basavanagoud B., Desai V.R., Patil S., $(\beta, \alpha)$-connectivity index of graphs, Appl. Math. Nonlinear Sci., 2017, 2(1), 21-30.

[22] Zhou S., Xu L., Xu Y., A sufficient condition for the existence of a k-factor excluding a given r-factor, Appl. Math. Nonlinear Sci., 2017, 2(1), 13-20.

[23] Petrović M., Aleksić T., Simić S., On the least eigenvalue of cacti, Linear Algebra Appl., 2011, 435, 2357-2364. 